\title{
Die integrative Kraft sozioökonomischer Bildung - oder: Herausforderungen für die sozialwissenschaftliche Kontextualisierung wirtschaftlicher Phänomene und ökonomischer Logiken
}

\author{
"engartner@soz.uni-frankfurt.de, Fachbereich Gesellschaftswissenschaften/Institut für Politikwissenschaft, Goethe-Universität \\ Frankfurt am Main
}

eingereicht am: 26.01.2019, akzeptiert am: 20.02.2019

\begin{abstract}
Die politischen, sozialen und ökonomischen Verwerfungen im Schatten der Wirtschafts- und Finanzkrise $2008 \mathrm{ff}$. verkennend, hält der Mainstream der Wirtschaftswissenschaften an seinen tradierten Modellen, Paradigmen und methodologischen Grundannahmen fest. Trotz leidenschaftlicher Debatten über die Notwendigkeit multi-, inter- und transdisziplinärer Zugänge in der Volkswirtschaftslehre halten sich viele wirtschaftswissenschaftliche Mythen unverändert. Monoparadigmatische Ansätze der orthodoxen Wirtschaftsdidaktik vernachlässigen kulturelle, historische, politische, ethische, gesellschaftliche und psychologische Einflüsse. Vor diesem Hintergrund soll der vorliegende Beitrag die erkenntnistheoretischen, bildungspolitischen und fachdidaktischen Mängel der neoklassisch geprägten Wirtschaftsdidaktik benennen, um sodann wissenschaftstheoretische Annahmen, fachdidaktische Prinzipien und bildungspolitische Implikationen einer sozioökonomischen Bildung zu skizzieren.
\end{abstract}

Keywords: sozioökonomische Bildung, Multidisziplinarität, Lebensweltorientierung, homo oeconomicus

\section{The integrative power of socio-economic education, or: the challenges for the socio-scientific contextualisation of economic phenomena and logic}

Disregarding the political, social and economic aftermath of the 2008 financial crisis, mainstream economics clings to traditional models, paradigms and methodological assumptions. Despite heartfelt calls for multi-, inter- and transdisciplinarity, economic myths still permeate the discipline. Mono-paradigmatic economic education neglects cultural, historic, political, ethical, societal and psychological impacts. The contribution identifies the epistemological, educational and didactical deficits of neo-classically influenced economic education and outlines the epistemological assumptions, didactic principles, and implications for education policy resulting from a shift towards socio-economic education.

Keywords: socio-economic education, multidisciplinarity, environmental orientation, homo oeconomicus

„Das Übergreifen von Märkten und marktorientiertem Denken auf Aspekte des Lebens, die bislang von Normen außerhalb des Marktes gesteuert wurden, ist eine der bedeutsamsten Entwicklungen unserer Zeit."

Michael J. Sandel (2012:14)

Der Einfluss ökonomischer Denkweisen hat in den vergangenen Jahrzehnten einen kontinuierlichen Bedeutungszuwachs erfahren, was sich beispielsweise in der geradezu einzigartigen Ausweitung betriebswirtschaftlicher Denkansätze auf das Bildungs-, Sozial-, Gesundheits- und Verkehrswesen widerspiegelt. So werden mit jeder Privatisierung gewichtige Instrumente zur Gestaltung einer demokratisch legitimierten bildungs-, sozial-, gesundheits- und umweltpolitischen Entwicklung aus der Hand gegeben, schränkt die Stärkung der Marktkräfte doch zugleich die staatlichen Regulations- und Kontrollmöglichkeiten 
ein. Immer häufiger werden ökonomische Kategorien, Paradigmen und method(olog)ische Grundannahmen in den Rang von „Realfiktionen“ erhoben, was zur Folge hat, dass die Deregulierung der Märkte, die Orientierung auf das Kosten-Nutzen-Kalkül oder die Idealisierung des rational handelnden Individuums zu nur noch selten hinterfragten Erklärungsansätzen geworden sind (vgl. weiterführend Engartner 2016).

Somit hat sich auch gesamtgesellschaftlich der Eindruck verfestigt, dass „ökonomisches Wissen und die Fähigkeit, selbstbestimmt in wechselnden ökonomischen Handlungssituationen agieren zu können, [eine] wesentliche Voraussetzung für die Teilhabe eines Menschen an der Gesellschaft" sind (Deutscher Lehrerverband 2000: 1). Zum Beispiel hat die eigenverantwortliche finanzielle Planung massiv an Bedeutung gewonnen, seitdem die Zuzahlungspflicht bei Arzneimitteln ausgeweitet, der Leistungskatalog der gesetzlichen Krankenkassen gekürzt und die Bismarck'sche Sozialversicherungsarchitektur mit der Einführung des „Drei-Säulen-Modells“, sowohl in Österreich als auch in Deutschland, als Instrumentarien der kapitalgedeckten Altersvorsorge aufgekündigt wurden. Zugleich haben grundlegende ökonomische Kenntnisse für die politische Willensbildung an Bedeutung gewonnen. $\mathrm{Ob}$ das Arbeitslosengeld gesenkt oder erhöht, die Pendlerpauschale gekürzt, die Budgets für Bildung umgeschichtet oder für kriselnde Banken milliardenschwere „Rettungsschirme“ aufgespannt werden - dahinter stehen stets politische Konzepte, für deren Verständnis ökonomisches Grundwissen unabdingbar ist.

\section{Ökonomisierung der Lebenswelten}

Der gesellschaftlich weithin akzeptierte Bedeutungszuwachs ökonomischen Denkens lässt sich unter dem Schlagwort „Ökonomisierung der Lebenswelten" subsumieren. Angesichts dieser Wirkmächtigkeit wirtschaftswissenschaftlicher Denk- und Erklärungsansätze sieht sich die sozioökonomische Bildung mit gewaltigen Herausforderungen konfrontiert. Sie hat sich u.a. damit auseinanderzusetzen, dass die (Re-)Strukturierung einer wachsenden Zahl von Gesellschaftsbereichen nach Markt-, Effizienz- und Konkurrenzkriterien mit einem vielfach tolerierten Verzicht auf Ziele einer kritisch-emanzipatorischen Bildung einhergeht. Zugleich muss sie verdeutlichen, inwieweit eine allein auf die neoklassische Standardökonomie fokussierte ökonomische Bildung verkennt, dass historische Entwicklungsstränge, politische Gestaltungsmöglichkeiten, gesellschaftliche Rahmenbedingungen und rechtliche Vorgaben für ökonomische Sachverhalte immens bedeutsam sind. Daher muss sozioökonomische Bildung die enge Verflechtung der Gegenstandsbereiche Politik, Wirtschaft und Gesellschaft akzentuieren und die der sozialwissenschaftlichen Trias aus Politik-, Wirtschafts- und Gesellschaftswissenschaft zugrundeliegenden Denkweisen, Kategorien und Methoden in einen systematischen Zusammenhang bringen, konzeptionell ordnen und wissenschaftlich durchdringen.

Nach einer aus sozialwissenschaftlicher Perspektive verengenden Auffassung der neoklassisch orientierten Wirtschaftsdidaktik soll sich ökonomische Bildung zwar auch an den Lebenssituationen der Adressaten und Adressatinnen orientieren, sowie die Strukturund Funktionsprinzipien der Wirtschaftsordnung erörtern; aber „das Theorie- und Methodenwissen der Ökonomik [als] domänenspezifische[s] Proprium für die ökonomische Bildung" soll weiterhin anerkannt werden (Loerwald 2007: 30). Sollen jedoch Brückenschläge zu sozialwissenschaftlichen Nachbardisziplinen vollzogen werden, müssen überdies auch $\mathrm{Ka}-$ tegorien ökonomischen Denkens Berücksichtigung finden, die von der orthodoxen Wirtschaftsdidaktik bislang nur unzureichend adressiert worden sind:

1. Ökonomisches Handeln vollzieht sich in einem engen Wechselverhältnis mit politischen Rahmenbedingungen, psychologischen Gesetzmäßigkeiten, gesellschaftlichen Grundwerten und geographischen Gegebenheiten.

2. Wirtschaftliches Handeln ist in der Mehrzahl der Fälle zweck- und zielbestimmt (Rationalitätsprinzip), wird aber nach Erkenntnissen der experimentellen Wirtschaftsforschung insbesondere auch von Fairness, Reziprozität und Vertrauen geprägt.

3. Wirtschaftlich handelnde Akteure und Akteurinnen treffen ihre Entscheidungen unter unsicheren Vorzeichen, d.h. sie haben aufgrund unvollkommener Informationen nur begrenzte Möglichkeiten der Voraussicht.

4. In ökonomisch geprägten Lebenssituationen vollziehen sich Handlungen typischerweise als Ausgleich konkurrierender Interessen in einem auf Wettbewerb angelegten System, das institutionell und regulatorisch eingebunden, d.h. ordnungspolitisch gestaltet ist oder gestaltet werden kann.

\section{Anspruch der lebensweltorientierten Multidisziplinarität}

Sollen wirtschaftliche Phänomene und Prozesse sowie ökonomische Denkweisen und Paradigmen gesellschaftswissenschaftlich kontextualisiert werden, bedarf es somit einer ergänzenden politik-, gesellschafts-, geschichts- und kulturwissenschaftlichen 
Perspektivierung. Schließlich ist das sozioökonomische Grundwissen „aus der Perspektive der Subjekte entlang relevanter sozioökonomisch geprägter Lebenssituationen zu ermitteln, in denen sie individuelle Entscheidungen treffen müssen, sowie entlang der Herausforderungen sozioökonomischer Entwicklungen, die sie beurteilen und mitgestalten können sollten" (Weber i. D.). Ausgangspunkt ist die Identifikation der realen Lebenswelten, „in denen private und gesellschaftliche Akteure handeln, die durch unterschiedliche Koordinationsformen in Beziehung zueinanderstehen, von unterschiedlichen Institutionen und Ordnungen gegenwärtig beeinflusst werden, die interdependent, aber auch gestaltbar sind“ (ebd.). Der der sozioökonomischen Bildung immanente (sozialwissenschaftlich) integrative Zugang lässt sich somit nicht nur wahrnehmungspsychologisch begründen, sondern auch aus den didaktischen Prinzipien der Situations-, Lebenswelt-, Problem- und Wissenschaftsorientierung ableiten. Ökonomisches zielt ebenso wie politisches Lernen auf Entscheidungs-, Reflexionsund Handlungsfähigkeit und orientiert sich mithin an dem sozialwissenschaftlich etablierten Bildungsziel der „Mündigkeit“ (vgl. weiterführend Hedtke 2014: 313).

Der Anspruch politisches, gesellschaftliches, historisches, kulturelles und ökonomisches Lernen miteinander zu verzahnen, leitet sich in erster Linie aus dem Gebot der Multidisziplinarität ab, welches wiederum auf der Annahme basiert, dass wir in einer von Pluralität geprägten Gesellschaft leben, in der die „Wirklichkeit [...] aus (unüberschaubar) vielen einzelnen Fakten, Dingen, Ideen besteht, die in sehr unterschiedlicher Weise zueinander in Beziehung stehen bzw. gesetzt werden können" (Schubert \& Klein 2011: 225). Sozioökonomisches Lernen erkennt aber nicht nur die gesellschaftliche Pluralität an, sondern auch die Vielgestaltigkeit sozialwissenschaftlicher Paradigmen, Theorien und Method(ologi)en. Schülerinnen und Schülern soll vermittelt werden, dass multidisziplinäre, -paradigmatische und -methodologische Zugänge die entscheidende Voraussetzung für die systematische Durchdringung sozialwissenschaftlicher Frage- und Problemstellungen darstellen. Obwohl Multiperspektivität zum Beispiel disziplinäre Perspektivität voraussetzt, lässt sich die gesellschaftliche, politische und ökonomische Wirklichkeit sachgerecht nicht nur aus einer Perspektive erfassen - jedenfalls dann nicht, wenn man sich dem Anspruch verpflichtet sieht, dass sozialwissenschaftliche Bildung auf die Entfaltung gesellschaftswissenschaftlicher Komplexität zielt (vgl. Engartner 2017). Die Bewusstseinsbildung bezüglich impliziter Annahmen über domänenspezifische Theorien, Methoden, Welt- und - dies gilt es zu betonen - Men- schenbilder sollte daher sowohl Grundlage als auch Ziel jedes kompetenzorientierten Unterrichts im Auftrag sozioökonomischer Bildung sein.

\section{Welches Menschenbild liegt der sozio- ökonomischen Bildung zugrunde?}

Unabhängig davon, welche didaktischen Prinzipien zur Auswahl geeigneter Themen für den sozioökonomischen Unterricht Anwendung finden, ist dieser Entscheidung eine Frage vorzuschalten, auf die es unzählige - und kaum als „richtig“ oder „falsch“ zu klassifizierende - Antworten gibt: Welches Menschenbild soll der sozioökonomischen Bildung zugrunde liegen? Der von den klassischen Ökonomen um Vilfredo Pareto, David Ricardo und John Stuart Mill skizzierte homo oeconomicus, der stets am Eigennutz orientierte, zweckrationale Entscheidungen trifft, galt lange Zeit als "Säulenheiliger" - insbesondere in Abgrenzung zum Menschenbild des homo sociologicus (vgl. Lindenberg 1990). Dieses idealtypische Bild ist jedoch ins Wanken geraten: „Der homo oeconomicus ist tot", titelte die Financial Times Deutschland am 14. März 2001 unter Verweis auf die Erkenntnisse der experimentellen Wirtschaftsforschung, d.h. konkret unter Bezugnahme auf die von Gary Bolton und Axel Ockenfels (2000) entwickelte ERC-Theorie, die unter der Trias „Equity, Reciprocity and Competition“ erläutert, dass Menschen je nach Kontext fair, wechselseitig kooperativ oder wettbewerbsorientiert entscheiden - und nicht durchweg egoistisch.

So sieht auch Armin Falk den homo oeconomicus, und die damit verbundene Annahme ökonomisches Verhalten wäre frei und unabhängig, durch den homo reciprocans ergänzt, der sich der Norm der Reziprozität verpflichtet fühlt (vgl. 2003: 166 ff.). Daraus folgert Birger P. Priddat eine Erweiterung von Verhaltensspektren um die Analyse von Heuristiken, also ,alle möglichen, selbstdefinierten Verhaltenstypen, die nicht nur auf Rationalität ausgelegt sind, sondern vielmehr auf einfache Entscheidungs- und Anschlussfähigkeit“ (2013: 136). Im Kontext der Sozioökonomik wird das Individuum folglich als sozial eingebettet, statt als atomistisch begriffen, was als weitreichende Differenz zur Mainstream-Ökonomik zu konstatieren ist (vgl. Davis 2011: 213). Individuen treten demnach nicht in sämtlichen Lebenslagen als egoistische Nutzenmaximierer/innen auf, sondern werden unter Umständen von sozialen, kulturellen und politischen Präferenzen geleitet. So kann aufgrund einer ausgeprägten Ungleichheitsaversion die Bereitschaft bestehen, materielle Verluste einzugehen, um eine egalitäre Verteilung herzustellen (vgl. Fehr \& Schmidt 2005). 
Wissenschaftstheoretische Inkonsistenz, mangelnde empirische Validität und eine daraus resultierende eingeschränkte Prognosefähigkeit haben die „SemiFiktion" des homo oeconomicus an Bedeutung verlieren lassen. Wenn das Modellkonstrukt aber nach den neuesten Erkenntnissen der Wirtschaftswissenschaft selbst im Kontext ökonomischer Sach- und Entscheidungslogiken kein letztgültiges Erklärungsmuster mehr liefert, muss dies unweigerlich Auswirkungen auf das in der sozioökonomischen Bildung zu vermittelnde Menschenbild haben. Die mit der Abkehr von der neoklassischen Standard- und Lehrbuchökonomie verbundene Einsicht, dass sich der Mensch erst als ein in der Gemeinschaft handelndes Wesen entfalten kann, zielt auf Handlungsmotive wie beispielsweise Dankbarkeit, Eitelkeit, Mitleid, Solidarität oder Umweltbewusstsein. Diese Einflussfaktoren, die zunehmend in ökonomische Theoriegebäude integriert werden, sollten in ein multiperspektivisches Menschenbild sozioökonomischer Prägung überführt werden. Nur wenn ökonomische Gesetzmäßigkeiten auf der Grundlage gesellschaftlicher Konnotationen in Bildungs- und Erziehungsprozesse eingebunden werden, wird der für Schulen konstitutive Allgemeinbildungsanspruch eingelöst.

\section{Ausrichtung von Lehr- und Lerninhalten in der sozioökonomischen Bildung}

In Zeiten, in denen immer mehr Gesellschaftsbereiche nach dem Vorbild des Marktes geordnet werden, ist eine vertiefte Auseinandersetzung mit dessen (Dys-) Funktionalitäten nicht nur sinnvoll, sondern geradezu unabdingbar. Überdies sollte im Kontext der sozioökonomischen Bildung Berücksichtigung finden, dass Märkte die Anwendung von Kategorien wie Gerechtigkeit, Solidarität und soziale Balance nach den herkömmlichen, zumeist noch immer in der Tradition der Neoklassik stehenden Modellen nicht zulassen. Insofern stellt Gerd Steffens eine wegweisende Frage: „Kann ökonomisches, also per definitionem auf individuelle Nutzenmaximierung gerichtetes Verhalten Gesellschaftlichkeit, soziale Synthese oder - bescheidener - Motive für den sozialen Zusammenhalt hervorbringen" (2008: 28)? Zu Recht wirft er die Frage auf, wie sich im Kontext sozialwissenschaftlicher Bildung Potenziale entwickeln lassen, „die die zerstörerische Kraft rein egoistischen Denkens bändigen, begrenzen, [und] womöglich in sozialen Zusammenhalt verwandeln“ (ebd.).

Bislang konzentriert sich der Mainstream der Wirtschaftswissenschaften unverändert auf die Knappheit von Gütern und die Notwendigkeit ökonomischer Dispositionen, sodass die kulturelle, historische und politische Dimension weitgehend ausgeblendet wird. „Dieser ,ökonomische Imperialismus', bei dem politische Handlungen und Motivationen dem Muster der rationalen Kosten-Nutzen-Überlegungen von Marktkontrahenten angeglichen werden, hat zwar in manchen Fällen einige neue Perspektiven und Einsichten geliefert, verfehlt aber vollkommen die - von der ,Alten Politischen Ökonomie' angestrebte - Aufgabe, die Besonderheiten sich wandelnder politischer, soziologischer und historischer Konstellationen zu berücksichtigen, die bei einer isolierten wirtschaftstheoretischen Sicht (...) vernachlässigt werden" (Rothschild 2004: 19). Aus dieser Haltung rührt ein Großteil der Skepsis gegenüber ökonomischen Lehr- und Lerninhalten, können diese doch sehr unterschiedlich definiert werden, zum Beispiel entweder eine dezidiert betriebs- oder eine eher volkswirtschaftliche Dimension aufweisen, wobei allerdings beide entweder mathematisch-modellhaft oder interdisziplinär sein können. Um die wechselseitigen Bezüge zwischen Politik, Soziologie und Ökonomie einerseits und ihre bisweilen unterschiedliche Logik andererseits zu erklären, sollten verstärkt Lehr- und Lerninhalte thematisiert werden, die eine Klammer zwischen diesen Sphären bieten.

\section{Konsum als beispielhaftes Feld sozio- ökonomischer Bildung}

Wie eine differenzierte Auseinandersetzung mit primär ökonomischen Themen im Kontext sozioökonomischer Bildung ausfallen kann, zeigt das uns täglich in den unterschiedlichsten Formen begegnende Phänomen des Konsums, weil es - anders als beispielsweise das neoklassische Gütermarktmodell oder das "Gefangenendilemma“ - unabhängig von Alter, Kenntnisstand und Zeitbudget zum Gegenstand des Unterrichts gemacht werden kann. Dadurch bedingt, dass der Wohlstandszuwachs die Einkommen der meisten Menschen gesteigert und insbesondere in den entwickelten Industriestaaten immer mehr von ihnen in den monetären Wirtschaftskreislauf einbezogen hat, kennzeichnet beinahe alle Bevölkerungsschichten eine bislang unbekannte Extensität materieller Möglichkeiten: „Dies hat zur Folge, dass es in unserer Gesellschaft so gut wie keinen mehr gibt, der nicht bereits im Kindes- und Jugendalter in das ihn umspannende Netz des Konsums auf vielfältige, verborgene oder manifeste Weise eingeflochten wäre" (Mertens 1998: 202), wobei Unterschiede in den Einkommen als Resultat einer neoliberalen Politik seit den 1990er Jahren die Schere zwischen Arm und Reich merkbar geöffnet und die Sicherung des eigenen Überlebens für manche zur ständigen Herausforderung gemacht hat. 
Wie (komplex) sich sozioökonomisches Denken darstellen kann, zeigt die Vielgestaltigkeit sozialwissenschaftlicher Konsumbildung. Eine rein wirtschaftswissenschaftliche Betrachtung von Konsum als Verbrauchshandlung vernachlässigt, zum Beispiel, seine soziale, emotionale, kulturelle und ästhetische Wirkmächtigkeit - und reduziert Konsumieren auf Finanzieren. Da Konsum Menschen prägt und Menschen Konsumangebote prägen (können), sollten die partizipatorischen Chancen, Risiken und Grenzen erörtert werden, wenn Konsumentinnen und Konsumenten ihre Rolle nutzen, um der Konsumgesellschaft ihren Stempel aufzudrücken. Die Entscheidung, ob ich trotz eines womöglich knappen Budgets (ethisch verantwortungsvoll) konsumiere, ist keineswegs trivial, sondern - im Gegenteil - in besonderer Weise komplex, wie die nachfolgenden Fragen zeigen: Vertraue ich der Reduktion von Informationen durch Anbieter von Gütesiegeln - und wenn ja, welchen? Schone ich die Umwelt mit einer mehrfach zu nutzenden Leinentasche oder erwerbe ich im Nachgang des Einkaufs eine Einweg-Plastiktüte an der Kasse? Spende ich für eine karitative Einrichtung oder spare ich das Geld für ein Konzert, bei dem ich ggf. eher den marktdominanten Ticketanbieter als die Band finanziere? Durch die Digitalisierung der Lebenswelten sind die Einflüsse über internetbasierte Informations-, Werbe-, Unterhaltungs-, Inszenierungs- und Vernetzungsmedien noch wirkmächtiger geworden, wie prominente YouTuberinnen und YouTuber mit ihren Social Commerce-Auftritten belegen.

Vor dem Hintergrund dieser Komplexität folgt sozioökonomische Konsumbildung einem integrativen sozialwissenschaftlichen Ansatz. In dessen Rahmen gilt es, die Risiken des Konsumismus zu identifizieren, die partizipatorischen Potenziale konsumbürgerschaftlichen Handelns zu entschlüsseln und die „Politisierung des Konsums“ (Lamla 2006) bzw. die „Moralisierung der Märkte“ (Stehr 2007) zu thematisieren. Dieser Zugang, wonach der „schlafende Riese Konsument" oder die Konsumentin erwachen und den „Kaufakt in eine Abstimmung über die weltpolitische Rolle der Konzerne" verwandeln soll, ist für eine sozioökonomische Perspektivierung unabdingbar (Beck 2002: 131). Daraus folgt, dass Konsumentinnen und Konsumenten ebenso wie Produzentinnen und Produzenten oftmals die an der reinen Nutzenund Wertvermehrung ausgerichtete Zweckrationalität überwinden und stattdessen ein von moralischen Kriterien geleitetes (Markt-)Verhalten praktizieren können sollten. Reflektiertes Konsumieren erfordert somit Analyse-, Urteils- und Handlungsfähigkeiten, die in handlungs- sowie problem- und konfliktorientierten pädagogischen Arrangements erprobt werden können. Dabei bietet gerade die Auseinandersetzung mit Konsumthemen die Chance für eine lebensweltnahe Beschäftigung mit Inhalten, die einen Brückenschlag zwischen Ökologie, Ökonomie und Politik erlauben.

\section{Didaktisch-methodische Säulen eines Konzepts sozioökonomischer Bildung}

Aufgrund der Fülle von Wirtschaftsthemen ist es unerlässlich, dem didaktischen Gebot der Exemplarität $\mathrm{zu}$ folgen, $d$. h. eine sach- und schüler/innengerechte Inhaltsauswahl zu treffen (vgl. weiterführend Engartner 2010). Ein wesentliches Auswahlkriterium ist darin zu sehen, dass die Lernenden anhand der gewählten Themen verallgemeinerungsfähige Strukturen und Funktionsweisen einer modernen, funktional ausdifferenzierten Gesellschaft entschlüsseln können. Damit sollen nicht nur klassische Kompetenzen ökonomischer Bildung gefördert werden, sondern insbesondere auch die politische Urteilsbildung. Konstitutive Merkmale der Sozioökonomiedidaktik sind - neben der für sämtliche Fachdidaktiken maßgeblichen Subjektorientierung - die Prinzipien der Problem- und der Konfliktorientierung. Die entsprechend getroffene Auswahl der jeweiligen Probleme, Konflikte oder Fälle sieht zum einen vor, dass sie als Lerngegenstände beispielhaft sind - insofern diese einen praxisbedeutsamen Ausschnitt der Wirklichkeit repräsentieren -, verlangt jedoch zugleich auch eine (subjektive) Bedeutsamkeit für die Lernenden, befördert dies doch im Allgemeinen die Lernmotivation. Insofern müssen die Themen nicht nur die Möglichkeit bieten, Grundsätzliches, Wesentliches, Strukturelles und Gesetzmäßiges zu erarbeiten, sondern auch an die gegenwärtige und zukünftige Lebenswelt der Zielgruppe anknüpfen.

Vorrangiges Ziel sozioökonomischer Bildung ist es, Lernende dazu zu befähigen, nach sachlich begründeten und persönlich definierten Kriterien analysieren, urteilen und handeln zu können. Analytisch muss dabei das Prinzip der Problemorientierung von einer an Konflikten orientierten Didaktik unterschieden werden: Während die Problemorientierung den Inhalt des Sozioökonomischen thematisiert und gesellschaftlich kollektiv definierte Probleme wie Arbeitslosigkeit, Wirtschaftskrise, Chancenungleichheit etc. fokussiert, betont die Konfliktorientierung politische, gesellschaftliche und ökonomische Prozesse, die von verschiedenen Akteuren beeinflusst und damit verändert werden können. Nicht zuletzt mit Blick auf die Dichotomie zwischen „den Schwachen“ und „den Starken" soll deutlich werden, dass es Regulierungen bedarf, um Konflikte zu schlichten, zu beschwichtigen oder gar nicht erst aufkeimen zu lassen. 
Anders als in der neoklassisch geprägten ökonomischen Bildung sollen im Rahmen der sozioökonomischen Bildung gesellschaftliche und persönliche Schlüsselprobleme akzentuiert werden: „Ökonomische Relevanz erhält die Problemorientierung dadurch, dass jegliches Wirtschaften, ob auf der individuellen oder der politischen Ebene, grundsätzlich vor einem Problem steht: Wie können die verfügbaren Mittel rational zur Erreichung von Zielen eingesetzt werden? Dabei wird die Problemlösung erschwert durch die Wahl der Ziele oder Mittel, durch existierende Zielkonflikte, Opportunitäts-kosten, Unsicherheiten und Risiken sowie durch nicht intendierte Folgen" (Weber 2008, S. 266).

Eine auf die Integration soziologischer und politikwissenschaftlicher Methoden und Paradigmen zielende sozioökonomische Bildung folgt unter methodologischen Gesichtspunkten den Prinzipien der Problemorientierung, der Multidisziplinarität und der Empirie, nimmt Schüler/innen- und Handlungsorientierung ernst und zeichnet sich durch eine pluralistische und wertoffene geistige Grundhaltung aus (Abb. 1):

\begin{tabular}{|c|c|}
\hline \multirow{2}{*}{ (1) Methodologie } & $\begin{array}{c}\text { problemorientiert } \\
\text { multidisziplinär } \\
\text { polyparadigmatisch } \\
\text { induktiv } \\
\text { empirisch }\end{array}$ \\
\hline (2) Perspektiven & $\begin{array}{c}\text { schülerorientiert } \\
\text { lebensweltorientiert } \\
\text { handlungsorientiert }\end{array}$ \\
\hline (3) Einstellungen & $\begin{array}{c}\text { wertoffen } \\
\text { pluralistisch } \\
\text { kritisch }\end{array}$ \\
\hline
\end{tabular}

Abb. 1: Prinzipien zur Integration der sozialwissenschaftlichen Teildisziplinen | in Anlehnung an: Hedtke 2012: 19

\section{Schlussfolgerungen}

Sollen wirtschaftliche Phänomene und Prozesse sowie ökonomische Denkweisen und Paradigmen gesellschaftswissenschaftlich kontextualisiert werden, bedarf es somit einer ergänzenden politik-, gesellschafts-, geschichts- und kulturwissenschaftlichen Perspektivierung. Ausgangspunkt der Lehr- / Lernprozesse muss die Identifikation der realen Lebenswelten sein, in denen Personen und Institutionen handeln. Lebenswelten sind jedoch nicht disziplinscharf $\mathrm{zu}$ trennen. Der der sozioökonomischen Bildung immanente (sozialwissenschaftlich) integrative Zugang lässt sich somit nicht nur wahrnehmungspsychologisch begründen, sondern auch aus den didaktischen Prinzipien der Situations-, Lebenswelt-, Problem- und Wissenschaftsorientierung ableiten. Ökonomisches zielt ebenso wie politisches Lernen auf Entscheidungs-, Reflexions- und Handlungsfähigkeit, orientiert sich mithin an dem sozialwissenschaftlich etablierten Bildungsziel der „Mündigkeit“.

Dabei muss gerade für die ökonomische Bildung die Auseinandersetzung mit wenigstens einer alternativen Position als pluralistisches Minimum benannt werden. Grundsätzlich müssen ökonomische Themen $\mathrm{zu}$ historischen Entwicklungssträngen, politischen Gestaltungsmöglichkeiten, gesellschaftlichen Rahmenbedingungen und rechtlichen Vorgaben in Bezug gesetzt werden damit das Prinzip der permanenten ethischen Reflexion ebenso gewahrt werden kann wie die sich auf den Pluralismus stützenden Prinzipien der Interdisziplinarität und Kontroversität (vgl. Graupe 2013; Exploring Economics 2019). Deshalb sollten im Einklang mit dem sozioökonomischen Ansatz die enge Verflechtung der Gegenstandsbereiche Politik, Ökonomie und Gesellschaft akzentuiert und die der sozialwissenschaftlichen Trias aus Politikwissenschaft, Ökonomie und Soziologie zugrundeliegenden Denkweisen, Kategorien und Methoden in einen systematischen Zusammenhang gebracht, konzeptionell geordnet sowie in einem sozialwissenschaftlichen Integrationsfach gefestigt werden.

\section{Literatur}

Beck, U. (2002): Macht und Gegenmacht im globalen Zeitalter. Neue weltpolitische Ökonomie. Suhrkamp, Frankfurt a. M.

Bolton, G. E. \& A. Ockenfels (2000): ERC: Theory of Equity, Reciprocity and Competition. In: American Economic Review 90(1). S. 166-193.

Davis, J. B. (2015): Die Konzeption des sozial eingebetteten Individuums. In: Hedtke, R. (Hrsg.): Was ist und wozu Sozioökonomie? Springer VS, Wiesbaden. S. 213-230.

Deutscher Lehrerverband (2000): Memorandum: Ökonomische Grundbildung ist Teil der Allgemeinbildung. http://www.lehrerverband.de/memoekon.htm (22.01.2019)

Engartner, T. (2010): Didaktik des Ökonomie- und Politikunterrichts. Schöningh - UTB, Paderborn/München/ Wien/Zürich.

Engartner, T. (2016): Staat im Ausverkauf. Privatisierung in Deutschland. Campus Verlag, Frankfurt a. M./New York.

Engartner, T. (2017): Politische und gesellschaftliche Schlüsselprobleme durch sozioökonomische Bildung er- 
schließen. In: Weber, B. (Hrsg.): Wirksamer Wirtschaftsunterricht. Schneider Verlag Hohengehren, Baltmannsweiler. S. 56-67.

Exploring Economics (2019): Orientieren. https://www. exploring-economics.org/de/ (12.02.2019)

Falk, A. (2003): Homo Oeconomicus versus Homo Reciprocans: Ansätze für ein neues wirtschaftspolitisches Leitbild? In: Perspektiven der Wirtschaftspolitik 4(1). S. 141-172.

Fehr, E. \& K. M. Schmidt (2005): The Economics of Fairness, Reciprocity and Altruism - Experimental Evidence and New Theories. (Discussion Paper, Bd. 20).

Graupe, S. (2013): Ökonomische Bildung. Die geistige Monokultur der Wirtschaftswissenschaft und ihre Alternativen. In: Coincidentia. Zeitschrift für europäische Geistesgeschichte 2. S. 139-165.

Hedtke, R. (2012): Die Wirtschaft in der Schule: Agendasetting, Akteure, Aktivitäten. Working Paper Nr. 3, Bielefeld.

Hedtke, R. (2014): Ökonomisches Lernen. In: Sander, W. (Hrsg.): Handbuch politische Bildung. Wochenschau Verlag, Schwalbach/Ts. S. 312-320.

Lamla, J. (2006): Politisierter Konsum - konsumierte Politik. Kritikmuster und Engagementformen im kulturellen Kapitalismus. In: Lamla, J. \& S. Neckel (Hrsg.): Politisierter Konsum - konsumierte Politik. Springer VS, Wiesbaden. S. 9-37.

Lindenberg, S. (1990): Homo Socio-oeconomicus: The Emergence of a General Model of Man in the Social Sciences. In: Journal of Institutional and Theoretical Economics 146(4). S. 727-748.
Loerwald, D. (2007): Ökonomische Bildung für bildungsferne Milieus. In: Aus Politik und Zeitgeschichte 57(32/33). S. 27-33.

Mertens, G. (1998): Umwelten. Eine humanökologische Pädagogik. Schöningh Verlag, Paderborn.

Priddat, B. P. (2013): Die neue Bevölkerung der Ökonomie. Multiple, faire, unwisssende und emotionale Akteure. In: Zeitschrift für Wirtschafts- und Unternehmensethik 2/2013. S. 136-156.

Rothschild, K. W. (2004): Die politischen Visionen großer Ökonomen. Stämpfli, Bern.

Sandel, M. J. (2012): Was man für Geld nicht kaufen kann. Ullstein, Berlin.

Schubert, K. \& M. Klein (2011): Das Politiklexikon. Bundeszentrale für politische Bildung, Bonn.

Steffens, G. (2008): Wirtschaftssubjekt und Staatsbürger. Konkurrierende Leitbilder und Konzepte politischer Bildung? In: Ders. \& B. Widmaier (Hrsg.): Politische und ökonomische Bildung. Konzepte - Leitbilder - Kontroversen. Hessische Landeszentrale für politische Bildung, Wiesbaden. S. 27-37.

Stehr, N. (2007): Die Moralisierung der Märkte. Eine Gesellschaftstheorie. Suhrkamp, Frankfurt a. M.

Weber, B. (2008): Problemorientierung, in: R. Hedtke/ dies. (Hrsg.): Wörterbuch ökonomische Bildung. Wochenschau Verlag, Schwalbach/Ts., 265-266.

Weber, Birgit (i. D.): Was ist relevantes sozioökonomisches Grundwissen? In: Autorengruppe Sozioökonomische Bildung (Hrsg.): Was ist gute ökonomische Bildung? Wochenschau Verlag, Frankfurt a. M. 